\title{
Electric-Field-Induced Polar Order and Localization of the Confined Electrons in $\mathrm{LaAlO}_{3} / \mathrm{SrTiO}_{3}$ Heterostructures
}

\author{
M. Rössle, ${ }^{1}$ K. W. Kim, ${ }^{1,2}$ A. Dubroka, ${ }^{1,3}$ P. Marsik, ${ }^{1}$ C. N. Wang, ${ }^{1}$ R. Jany, ${ }^{4}$ C. Richter, ${ }^{4,5}$ J. Mannhart, ${ }^{5}$ C. W. Schneider, ${ }^{6}$ \\ A. Frano, ${ }^{5,7}$ P. Wochner, ${ }^{8}$ Y. Lu, ${ }^{5}$ B. Keimer, ${ }^{5}$ D. K. Shukla, ${ }^{9}$ J. Strempfer, ${ }^{9}$ and C. Bernhard ${ }^{1, *}$ \\ ${ }^{1}$ University of Fribourg, Department of Physics and Fribourg Center for Nanomaterials, \\ Chemin du Musée 3, CH-1700 Fribourg, Switzerland \\ ${ }^{2}$ Department of Physics, Chungbuk National University, Cheongju 361-763, Korea \\ ${ }^{3}$ Department of Condensed Matter Physics, Faculty of Science, Masaryk University and Central European Institute of Technology, \\ Kotlár̆ská 2, CZ-61137 Brno, Czech Republic \\ ${ }^{4}$ Experimental Physics VI, Center for Electronic Correlations and Magnetism, Institute of Physics, \\ University of Augsburg, D-86135 Augsburg, Germany \\ ${ }^{5}$ Max-Planck-Institut für Festkörperforschung, Heisenbergstrasse 1, D-70569 Stuttgart, Germany \\ ${ }^{6}$ Paul Scherrer Institut, CH-5232 Villigen, Switzerland \\ ${ }^{7}$ Helmholtz-Zentrum Berlin für Materialien und Energie, Albert-Einstein-Strasse 15, D-12489 Berlin, Germany \\ ${ }^{8}$ Max-Planck-Institut für Intelligente Systeme, Heisenbergstrasse 3, D-70569 Stuttgart, Germany \\ ${ }^{9}$ Deutsches Elektronen-Synchrotron DESY, Notkestrasse 85, D-22603 Hamburg, Germany
}

(Received 17 September 2012; published 27 March 2013)

\begin{abstract}
With ellipsometry, x-ray diffraction, and resistance measurements we investigated the electric-field effect on the confined electrons at the $\mathrm{LaAlO}_{3} / \mathrm{SrTiO}_{3}$ interface. We obtained evidence that the localization of the electrons at negative gate voltage is induced, or at least enhanced, by a polar phase transition in $\mathrm{SrTiO}_{3}$ which strongly reduces the lattice polarizability and the subsequent screening. In particular, we show that the charge localization and the polar order of $\mathrm{SrTiO}_{3}$ both develop below $\sim 50 \mathrm{~K}$ and exhibit similar, unipolar hysteresis loops as a function of the gate voltage.
\end{abstract}

DOI: 10.1103/PhysRevLett.110.136805

PACS numbers: 73.20.- r, 78.20.-e, 77.70.+a

The mobile electrons at the interface between $\mathrm{TiO}_{2}$ terminated $\mathrm{SrTiO}_{3}$ (STO) substrates and thin $\mathrm{LaAlO}_{3}$ (LAO) layers are the subject of intense research efforts [1]. The confined electrons at the LAO/STO interface develop rather suddenly when the LAO layer reaches a minimal thickness of $n=4$ unit cells [2]. While a mixing of cations [3] or oxygen vacancies [4] may also contribute, the main origin of the confined electrons seems to be a charge transfer from the top surface of LAO to the LAO/STO interface [2,5]. The latter is caused by the polarity mismatch at the LAO/STO interface, which yields an electrostatic potential that increases with the LAO thickness [1]. The conductivity of the confined electrons can be readily modified with a gate voltage that enables the design of efficient field-effect devices [2]. For LAO/STO with $n=3$ that is still insulating, a metallic state can be induced with moderate electric fields [2]. Even the biased tip of an atomic force microscope has been used to write and erase conducting pathways [6]. For samples with $n \geq 4$, the conductivity can also be modified with a gate voltage, although an insulating state develops only at low temperature [7]. The confined electrons even develop a superconducting phase below $T_{c} \sim 0.2-0.4 \mathrm{~K}$ [8], which can also be suppressed with an electric field that induces a localized state [9]. The mechanism by which the external electric field induces the localization of the confined electrons is still under discussion [7,10-12]. In this Letter we present infrared (IR) ellipsometry, x-ray diffraction, and resistance measurements that reveal that a polar phase transition in $\mathrm{SrTiO}_{3}$ is playing an important role.

The LAO/STO heterostructures with $n=3$ (LS-3_1 and LS-3_2) and $n=5.5$ (LS-5.5_1 and LS-5.5_2) monolayers of LAO have been prepared as described in Ref. [2] and are detailed in the Supplemental Material [13]. A sketch of the contact arrangement and the polarity of the gate voltage is shown in Fig. 1(a). A $\mathrm{SrTi}^{18} \mathrm{O}_{3}$ (ST18O) crystal with a ferroelectric transition of $T_{\text {Curie }}=23 \mathrm{~K}$ has been obtained by placing a $\mathrm{SrTi}^{16} \mathrm{O}_{3}$ substrate (ST16O) for a week at $1100{ }^{\circ} \mathrm{C}$ in an oxygen atmosphere with $92 \%{ }^{18} \mathrm{O}$. This resulted in the exchange of ${ }^{16} \mathrm{O}$ with ${ }^{18} \mathrm{O}$ into a depth of approximately $100 \mu \mathrm{m}$ with an efficiency of $\sim 90 \%$ as confirmed by the redshift of the Raman- and IR-active phonon modes.

For IR ellipsometry we used a homemade setup attached to a fast Fourier-transform interferometer (Bruker 113v) as described in Ref. [14]. In the terahertz range the ellipsometry was performed with a homemade setup similar to that in Ref. [15]. The modeling of the ellipsometry data was done with the Woollam VASE software [16]. The X-ray diffraction data were obtained at beam line P09/PETRA III at DESY, Hamburg, Germany, at a photon energy of $9.8 \mathrm{keV}$ with a closed cycle cryostat mounted on a sixcircle diffractometer and an avalanche photodetector with a high dynamic range. The two-point resistance measurements were performed with a Quantum Design Physical Properties Measurement System. 

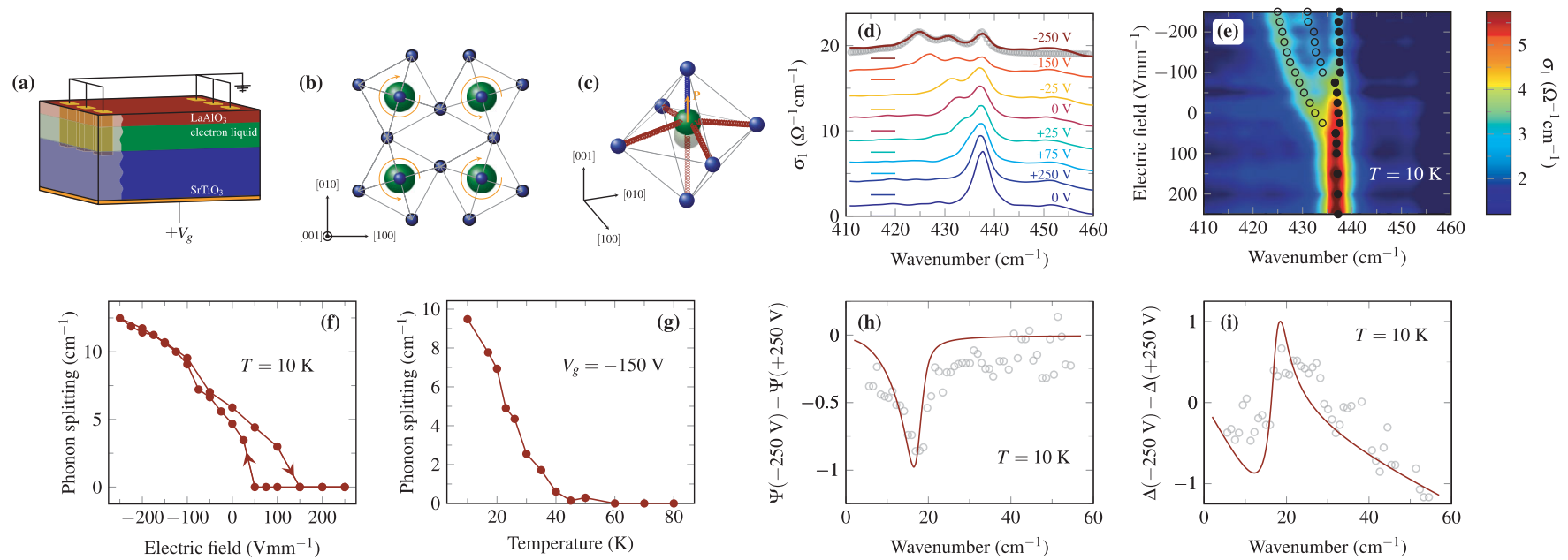

FIG. 1 (color). (a) Sketch of a LAO/STO structure and the electrodes used to apply backgate voltages up to $\pm 250 \mathrm{~V}$. (b) Antiphase rotations of the $\mathrm{TiO}_{6}$ octahedra that gives rise to the IR-active $R$ mode at $438 \mathrm{~cm}^{-1}$ of tetragonal $\mathrm{SrTiO}_{3}$. (c) Polar displacement of the $\mathrm{Ti}$ ions along the $[001]_{c}$ axis showing the effect on the length and the eigenfrequency of the TiO bonds as discussed in the text. (d), (e) IR spectra at $10 \mathrm{~K}$ showing the voltage dependent splitting of the $R$ mode in LS-5.5_1. Solid lines in (d) show the $y$ shift that has been added for clarity. Solid and open circles in (e) mark the splitting of the $R$ mode as discussed in the text. (f) Electric-field dependence of the maximal splitting of the $R$ mode. (g) $T$ dependence of the $R$-mode splitting. (h), (i) Difference spectra of the ellipsometric angles $\Psi(-250 \mathrm{~V})-\Psi(+250 \mathrm{~V})$ and $\Delta(-250 \mathrm{~V})-\Delta(+250 \mathrm{~V})$ measured at $10 \mathrm{~K}$ on LS-5.5_2 (open symbols) and model calculations (solid lines).

Some of us have previously shown that with IR ellipsometry one can study the mobility and even the density depth profile of the confined electrons of LAO/STO heterostructures with $n \geq 4$ [17]. Subsequently, we wanted to investigate the electric-field effect on the confined electrons. To our surprise, when studying LS-5.5_1, we observed some pronounced changes of the IR-active phonon modes that are suggestive of a polar phase transition in a fairly thick STO layer at the LAO/STO interface.

Figures 1(d) and 1(e) display the IR spectra at $10 \mathrm{~K}$, which reveal an anomalous, electric-field-induced splitting of the mode at $438 \mathrm{~cm}^{-1}$. This so-called $R$ mode involves an antiphase rotation of the neighboring $\mathrm{TiO}_{6}$ octahedra as shown in Fig. 1(b). It originates from the $R$ point at the boundary of the cubic Brillouin zone and becomes weakly IR active in the tetragonal state below $105 \mathrm{~K}[18,19]$ where the antiferrodistortive phase transition leads to a doubling of the unit cell and thus a backfolding of the Brillouin zone [20,21].

This $R$ mode is hardly affected as $V_{g}$ is initially increased from 0 to $+250 \mathrm{~V}$, whereas it starts to develop two side peaks at lower frequency when $V_{g}$ is subsequently decreased to $-250 \mathrm{~V}$. Figure 1(f) details how this splitting evolves with $V_{g}$ and highlights its unipolar and hysteretic behavior. Figure $1(\mathrm{~g})$ displays the $T$ dependence of the splitting at $V_{g}=-150 \mathrm{~V}$, which develops below $50 \mathrm{~K}$ in the vicinity of the avoided phase transition of the incipient ferroelectric $\mathrm{SrTiO}_{3}$ [22].

Figures 2(a) and 2(b) show that a strikingly similar splitting of the $R$ mode occurs in the oxygen-isotopeexchanged ST18O single crystal in the ferroelectric state below $T_{\text {Curie }} \approx 23 \mathrm{~K}[23,24]$. Figures 2 (c) and 2(d) confirm that the mode splitting is absent in ST16O, which remains in a so-called quantum paraelectric state for which the oxygen-isotope-induced enhancement of the lattice quantum fluctuations suppresses the ferroelectric transition [25]. In the latter, no sign of an anomaly is observed despite two additional, nonpolar structural phase transitions that further reduce the lattice symmetry below $105 \mathrm{~K}[18,26]$.

The $R$-mode splitting is indeed well understood in terms of a polar displacement of the Ti ions away from the center of the $\mathrm{TiO}_{6}$ octahedra. Figure 1(c) shows a sketch of
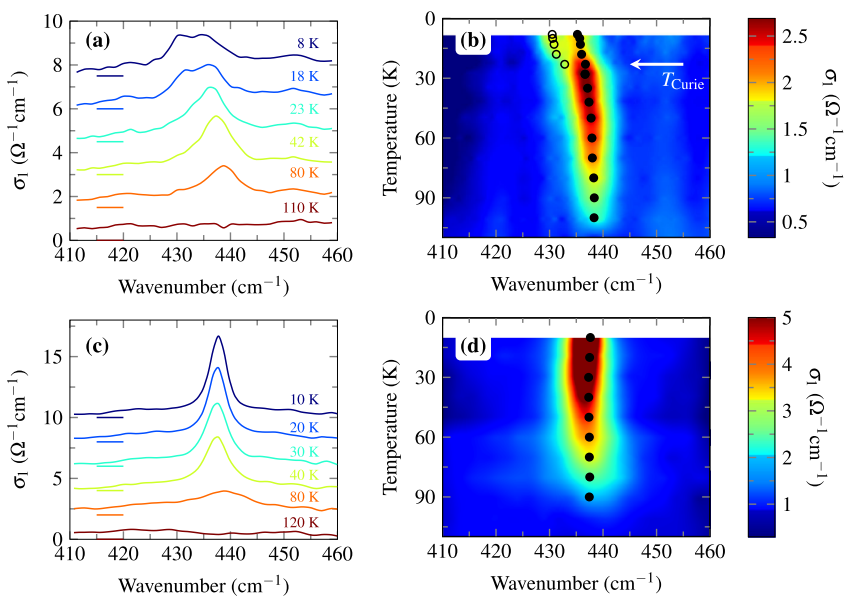

FIG. 2 (color online). (a), (b) IR spectra showing the $T$ dependence of the $R$ mode in a $\mathrm{SrTi}^{18} \mathrm{O}_{3}$ single crystal with a ferroelectric transition at $T_{\text {Curie }}=23 \mathrm{~K}$. (c), (d) Corresponding spectra of quantum paraelectric $\mathrm{SrTi}^{16} \mathrm{O}_{3}$. 
(exaggerated) displacement along the $[001]_{c}$ direction (in cubic notation). The colored springs indicate the redshift and blueshift of the eigenfrequency of the Ti-O bonds that are caused by the respective increase or decrease of the bond lengths. This illustrates that the polar displacement gives rise to an anisotropic softening and thus to a splitting of the $R$ mode. The largest softening occurs when the light polarization is perpendicular to the polar displacement. The alternative case of a displacement along $[110]_{c}$ or $[111]_{c}$ is discussed in the Supplemental Material [13].

The data in Figs. 1(d) and 1(e) thus are well explained by a field-induced, polar layer in the vicinity of the LAO/STO interface. The original peak at $438 \mathrm{~cm}^{-1}$, whose intensity decreases while the position remains unchanged, is assigned to the response of the paraelectric bulk of the ST16O substrate. The two additional modes originate from the polar STO layer at the LAO/STO interface. The grey circles in Fig. 1(d) show a simulation of the spectrum at $V_{g}=-250 \mathrm{~V}$ assuming a $1 \mu \mathrm{m}$ thick polar STO layer for which the position, width, and relative strength of the $R$ modes have been adjusted while the other parameters have been obtained from the spectrum at $+250 \mathrm{~V}$ as described in the Supplemental Material [13].

Further evidence for the polar nature of this transition has also been obtained from the electric-field dependence of the so-called soft mode, which directly involves the Ti-O displacement that gives rise to the ferroelectric order in STO. In paraelectric ST16O its eigenfrequency decreases from $\sim 100 \mathrm{~cm}^{-1}$ at $300 \mathrm{~K}$ to $\sim 15 \mathrm{~cm}^{-1}$ at $10 \mathrm{~K}$ [27-29]. In the ferroelectric state, this soft mode is known to exhibit a characteristic hardening as shown in in the Supplemental Material [13] for the case of ST18O. According to the Lyddane-Sachs-Teller relation, this hardening of the soft mode is directly related to the decrease of the dielectric constant. The $\mathrm{THz}$ ellipsometry data on LS-5.5_2 in Figs. 1(g) and 1(h) confirm that such a characteristic hardening of the soft mode does indeed take place. The effect is displayed in terms of the difference spectra of the ellipsometric angles, $\Psi(-250 \mathrm{~V})-\Psi(+250 \mathrm{~V})$ and $\Delta(-250 \mathrm{~V})-\Delta(+250 \mathrm{~V})$ (open symbols) where it gives rise to characteristic dip and resonance features. These are both well reproduced by a model calculation (solid lines) for a $1 \mu \mathrm{m}$ thick polar layer in which the soft mode hardening at $V_{g}=-250 \mathrm{~V}$ amounts to $1.8 \mathrm{~cm}^{-1}$. Further details and a discussion of the electric-field effect on the corresponding longitudinal mode are given in the Supplemental Material [13].

We emphasize that these characteristic phonon anomalies are difficult to understand in terms of a nonpolar distortion. For example, the gradual splitting of the $R$ modes cannot arise from a further increase of the unitcell size and a subsequent backfolding of an IR-active phonon branch from the Brillouin-zone boundary. In this case, the frequency of the new modes (and thus the mode splitting) would be constant, only their intensity would increase with the distortion. The magnitude of the $R$-mode splitting of $12 \mathrm{~cm}^{-1}$ at $V_{g}=-250 \mathrm{~V}$ is also difficult to reconcile with a nonpolar distortion that reduces the tetragonal symmetry. It amounts to a $2.8 \%$ reduction of the eigenfrequency $\omega$, which should result in a change of the Ti-O bond length, $d_{\text {Ti-O }}$, of $\sim 1 \%$ (because $\omega \propto 1 / d^{2}$ [30]). For the polar distortion the lattice constant is hardly affected because one Ti-O bond is shortened and the other expanded. However, for nonpolar and thus symmetric distortions this would result in a $1 \%$ change of the lattice constant that is not compatible with our x-ray diffraction data

Figures 3(a)-3(d) display $k$-space maps around the (002) Bragg peak of $\mathrm{SrTiO}_{3}$ at different gate voltages and temperatures. These data and additional scans around the (103) and $\left(\frac{\overline{3}}{2} \frac{1}{2} \frac{3}{2}\right)$ Bragg peaks (not shown) are not compatible with the shift of a significant part of the main Bragg peak by about 0.01 r.l.u. (where r.l.u. is reciprocal lattice units) that would be expected for nonpolar distortions as discussed above. The $k$-space maps show instead that two new satellite peaks develop at $T=10 \mathrm{~K}$ and $V_{g}=-250 \mathrm{~V}$. These are characteristic signatures of a domain state that develops in response to the polar order. Their spacing of $\Delta Q=0.0063$ r.l.u. with respect to (002) signifies a structural modulation with a period of $\sim 60 \mathrm{~nm}$ parallel to the $\mathrm{LAO} /$ STO interface with a preferred $[110]_{c}$ direction. It was previously shown $[31,32]$ that such superlattice peaks
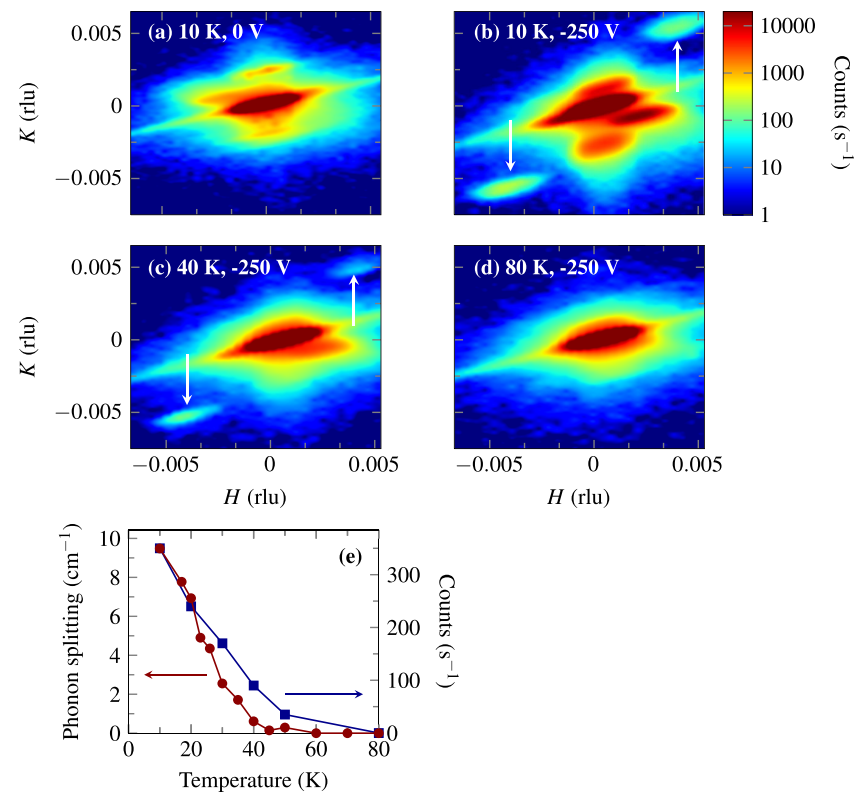

FIG. 3 (color online). Field-induced satellite Bragg peaks in the polar domain state of LS-5.5_1. (a), (b) Electric-field dependence of the $k$-space map around the (002) Bragg peak of ST16O at $T=10 \mathrm{~K}$. White arrows mark two new satellite peaks that appear at $V_{g}=-250 \mathrm{~V}$ at $(\bar{h} \bar{k} 2)$ and $(h k 2)$, where $h=0.004$ and $k=0.005$. (c)-(e) $T$ dependence of the satellite peaks at $V_{g}=-250 \mathrm{~V}$ in comparison with the $R$-mode splitting observed at $V_{g}=-150 \mathrm{~V}$. 
typically occur in ferroelectric thin films where they arise from antiphase stripe domains with an alternating polarization direction. In LAO/STO, the normal component of the unipolar order likely remains parallel to the applied electric field. The satellite peaks thus may arise from an alternation of the in-plane component of a tilted polarization or else from a modulation of the amplitude of the normal component. The observed domain size of $\sim 60 \mathrm{~nm}$ at a layer thickness of $1 \mu \mathrm{m}$ is roughly consistent with the trend reported in Ref. [32]. Figures 3(c)-3(e) show the $T$ dependence of the satellite peaks that disappear around $50 \mathrm{~K}$, similar to the splitting of the $R$ mode.

This raises questions about the origin of this electricfield-induced unipolar order and its relationship to the localization of the confined electrons. $\mathrm{SrTiO}_{3}$ is an incipient ferroelectric material for which the correlation length starts to diverge below $\sim 50 \mathrm{~K}$ but long range order is inhibited by the quantum lattice fluctuations [22,25]. Despite the thickness of the polar layer of $\sim 1 \mu \mathrm{m}$ at $-250 \mathrm{~V}$, the polar order thus may originate from the LAO/STO interface. A built-in electric field that is pointing toward the STO substrate likely plays an important role. It arises from the electronic and structural reconstruction at the interface and yields the band bending and the potential that confines the mobile electrons. Such a local field accounts for the unipolar nature of the order and explains its onset at a positive gate voltage [see Fig. 1(f)]. A corresponding lattice polarization in the first few STO monolayers has been predicted $[33,34]$ and observed with surface x-ray diffraction [35] and transmission electron microscopy [36]. The lattice distortion and the underlying local electric field were shown to increase as the LAO thickness grows from $n=2$ to 5 [35]. In good agreement, we find that the field-induced splitting of the $R$ mode is significantly smaller for LS-3_1 and is absent in a control device for which a thin Ti electrode is deposited directly on $\mathrm{SrTiO}_{3}$ (for details see the Supplemental Material [13]).

It seems unlikely that the confined electrons are playing an active role in the polar phase transition. The polarity of the electric order yields a negative ionic charge density at the LAO/STO interface for which the interfacial electrons cannot stabilize the order by screening the external electric stray field. On the other hand, the field-induced metal-toinsulator transition of the confined electrons may well be triggered or even driven by the development of the polar order. The latter reduces the polarizability of the lattice and thus its ability to screen defects that eventually localize the confined electrons.

Figure 4 reveals that the formation of the polar order and the rapid increase of the resistance of the LAO/STO heterostructure are indeed correlated. Figures 4(a) and 4(b) show that the voltage dependence of the resistance changes around $50 \mathrm{~K}$. At higher $T$ the resistance exhibits a weak, linear increase toward negative $V_{g}$. Below $50 \mathrm{~K}$, the resistance starts to increase much more rapidly and exhibits a
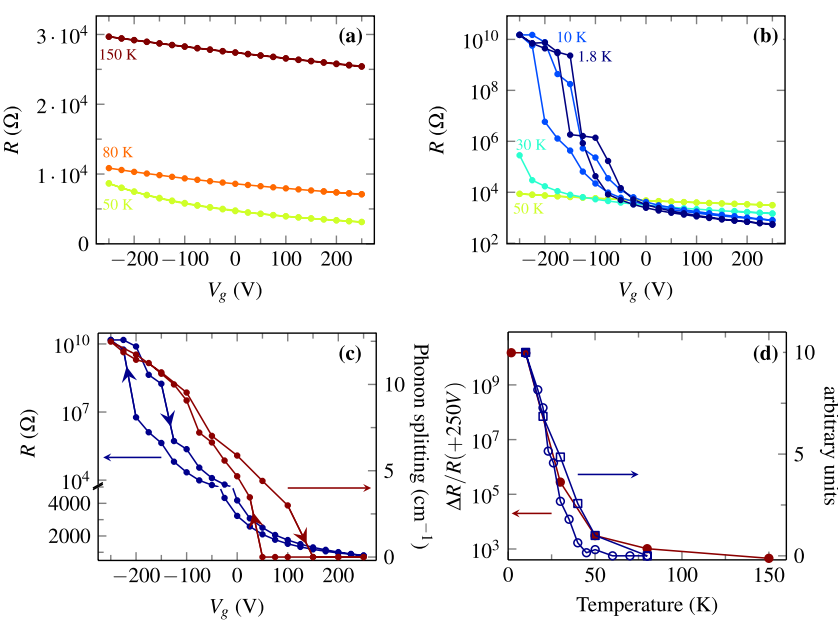

FIG. 4 (color online). Resistance versus gate voltage determined with a two-point measurement. (a), (b) $R$ - $V$ curves showing the change from a metallic regime at 80 and $150 \mathrm{~K}$ to the localization regime below $50 \mathrm{~K}$. (c) Comparison of the voltage loops at $10 \mathrm{~K}$ on LS-5.5_1, the resistance, and the splitting of the $R$ mode. (d) $T$ dependence of the field-induced increase in resistance, $R(-250 \mathrm{~V})-R(+250 \mathrm{~V}) / R(+250 \mathrm{~V})$ (solid circles), the $R$-mode splitting (open circles), and the intensity of the x-ray satellite peaks (open squares).

hysteretic behavior. Figure 4(c) compares the voltage loops of the resistance at $10 \mathrm{~K}$ with the splitting of the $R$ mode as a measure of the polar order. It confirms that the fieldinduced electron localization occurs in close vicinity to the polar transition. Finally, Fig. 4(d) shows that the charge localization also develops below $50 \mathrm{~K}$. This comparison suggests that the field-induced localization of the confined electrons is strongly influenced or likely even directly induced by the polar order.

In conclusion, we obtained evidence that the electricfield-induced localization of the confined electrons in $\mathrm{LAO} / \mathrm{STO}$ heterostructures is induced, or at least strongly enhanced, by a polar phase transition in a fairly thick $(\sim 1 \mu \mathrm{m}) \mathrm{SrTiO}_{3}$ layer at the $\mathrm{LAO} / \mathrm{STO}$ interface. In particular, we showed that the charge localization and the polar order occur in the same voltage and temperature ranges and exhibit similar, unipolar hysteresis loops. Our findings suggest that this polar order and the subsequent reduction of the Coulomb screening need to be considered for the interpretation of electric-field-induced localization transition of the confined electrons.

This work is supported by the Schweizer Nationalfonds (SNF) Grants No. 200020-119784 and No. 200020140225, by the NCCR MaNEP, by the German Science Foundation (TRR80), and the CEITEC by CZ.1.05/1.1.00/ 02.0068. We appreciate the help of K. Conder and the support of Th. Lippert in preparing the isotope exchanged $\mathrm{SrTi}^{18} \mathrm{O}_{3}$ crystal. We acknowledge stimulating discussions with Ulrich Aschauer, Dionys Baeriswyl, Josef Humlíček, Andy Millis, Andrei Sirenko, Nicola Spaldin, and JeanMarc Triscone. 
*christian.bernhard@unifr.ch

[1] A. Ohtomo and H. Y. Hwang, Nature (London) 427, 423 (2004).

[2] S. Thiel, G. Hammerl, A. Schmehl, C. W. Schneider, and J. Mannhart, Science 313, 1942 (2006).

[3] P. R. Willmott, S. A. Pauli, R. Herger, C. M. Schlepütz, D. Martoccia, B. D. Patterson, B. Delley, R. Clarke, D. Kumah, C. Cionca, and Y. Yacoby, Phys. Rev. Lett. 99, 155502 (2007).

[4] A. Kalabukhov, R. Gunnarsson, J. Börjesson, E. Olsson, T. Claeson, and D. Winkler, Phys. Rev. B 75, 121404 (2007).

[5] N. Nakagawa, H. Y. Hwang, and D. A. Muller, Nat. Mater. 5, 204 (2006).

[6] C. Cen, S. Thiel, J. Mannhart, and J. Levy, Science 323, 1026 (2009).

[7] Y.C. Liao, T. Kopp, C. Richter, A. Rosch, and J. Mannhart, Phys. Rev. B 83, 075402 (2011).

[8] N. Reyren, S. Thiel, A. D. Caviglia, L. F. Kourkoutis, G. Hammerl, C. Richter, C.W. Schneider, T. Kopp, A. S. Ruetschi, D. Jaccard, M. Gabay, D. A. Muller, J. M. Triscone, and J. Mannhart, Science 317, 1196 (2007).

[9] A. D. Caviglia, S. Gariglio, N. Reyren, D. Jaccard, T. Schneider, M. Gabay, S. Thiel, G. Hammerl, J. Mannhart, and J. M. Triscone, Nature (London) 456, 624 (2008).

[10] C. Bell, S. Harashima, Y. Kozuka, M. Kim, B. G. Kim, Y. Hikita, and H. Y. Hwang, Phys. Rev. Lett. 103, 226802 (2009).

[11] A. D. Caviglia, M. Gabay, S. Gariglio, N. Reyren, C. Cancellieri, and J.-M. Triscone, Phys. Rev. Lett. 104, 126803 (2010).

[12] J. Biscaras, S. Hurand, C. Feuillet-Palma, A. Rastogi, R. C. Budhani, N. Reyren, E. Lesne, D. LeBoeuf, C. Proust, J. Lesueur, and N. Bergeal, arXiv:1206.1198.

[13] See Supplemental Material at http://link.aps.org/ supplemental/10.1103/PhysRevLett.110.136805 for typical ellipsometry spectra for $\mathrm{SrTiO}_{3}$ and the field-effect in the sample LS-3_1; a detailed explanation of the fitting procedure; and a discussion of the electric-field induced anisotropy of the longitudinal optical mode.

[14] C. Bernhard, J. Humlícek, and B. Keimer, Thin Solid Films 455-456, 143 (2004).

[15] N. Matsumoto, T. Hosokura, T. Nagashima, and M. Hangyo, Opt. Lett. 36, 265 (2011).

[16] http://www.jawoollam.com.
[17] A. Dubroka, M. Rössle, K. W. Kim, V. K. Malik, L. Schultz, S. Thiel, C.W. Schneider, J. Mannhart, G. Herranz, O. Copie, M. Bibes, A. Barthélémy, and C. Bernhard, Phys. Rev. Lett. 104, 156807 (2010).

[18] F. W. Lytle, J. Appl. Phys. 35, 2212 (1964).

[19] R. Loetzsch, A. Lubcke, I. Uschmann, E. Forster, V. Grosse, M. Thuerk, T. Koettig, F. Schmidl, and P. Seidel, Appl. Phys. Lett. 96, 071901 (2010).

[20] P. A. Fleury, J. F. Scott, and J. M. Worlock, Phys. Rev. Lett. 21, 16 (1968).

[21] J. Petzelt, T. Ostapchuk, I. Gregora, I. Rychetsky, S. Hoffmann-Eifert, A. V. Pronin, Y. Yuzyuk, B. Gorshunov, S. Kamba, V. Bovtun, J. Pokorny, M. Savinov, V. Porokhonskyy, D. Rafaja, P. Vanek, A. Almeida, M. R. Chaves, A. A. Volkov, M. Dressel, and R. Waser, Phys. Rev. B 64, 184111 (2001).

[22] E. Tosatti and R. Martonák, Solid State Commun. 92, 167 (1994).

[23] M. Itoh, R. Wang, Y. Inaguma, T. Yamaguchi, Y.-J. Shan, and T. Nakamura, Phys. Rev. Lett. 82, 3540 (1999).

[24] M. Yamaguchi, T. Yagi, R. Wang, and M. Itoh, Phys. Rev. B 63, 172102 (2001).

[25] K. A. Müller and H. Burkard, Phys. Rev. B 19, 3593 (1979).

[26] R. Blinc, B. Zalar, V. V. Laguta, and M. Itoh, Phys. Rev. Lett. 94, 147601 (2005).

[27] W. Cochran, Adv. Phys. 9, 387 (1960).

[28] A. S. Barker and M. Tinkham, Phys. Rev. 125, 1527 (1962).

[29] H. Vogt, Phys. Rev. B 51, 8046 (1995).

[30] L. Artus and J. Pascual, Phys. Condens. Matter 4, 5835 (1992).

[31] S. K. Streiffer, J. A. Eastman, D. D. Fong, C. Thompson, A. Munkholm, M. V. Ramana Murty, O. Auciello, G. R. Bai, and G. B. Stephenson, Phys. Rev. Lett. 89, 067601 (2002).

[32] D. D. Fong, G. B. Stephenson, S. K. Streiffer, J. A. Eastman, O. Auciello, P.H. Fuoss, and C. Thompson, Science 304, 1650 (2004).

[33] R. Pentcheva and W.E. Pickett, Phys. Rev. Lett. 102, 107602 (2009).

[34] U. Schwingenschlögl and C. Schuster, Europhys. Lett. 86, 27005 (2009).

[35] S. A. Pauli, S. J. Leake, B. Delley, M. Björck, C. W. Schneider, C. M. Schlepütz, D. Martoccia, S. Paetel, J. Mannhart, and P.R. Willmott, Phys. Rev. Lett. 106, 036101 (2011).

[36] C. L. Jia, S. B. Mi, M. Faley, U. Poppe, J. Schubert, and K. Urban, Phys. Rev. B 79, 081405 (2009). 